\title{
Substitutions and Möbius disjointness
}

\author{
S. Ferenczi \\ J. Kułaga-Przymus* \\ M. Lemańczyk* \\ C. Mauduit
}

August 24, 2018

\begin{abstract}
We show that Sarnak's conjecture on Möbius disjointness holds for all subshifts given by bijective substitutions and some other similar dynamical systems, e.g. those generated by Rudin-Shapiro type sequences.
\end{abstract}

\section{Contents}

1 Introduction 2

2 Basic tools

2.1 Spectral theory ......................... 5

2.2 Joinings ............................ 5

2.3 Compact group extensions . . . . . . . . . . . . . . . . . . . 6 6

2.4 Generic points .............................. 7

3 Basic objects 7

3.1 Odometers, Morse cocycles and Toeplitz extensions . . . . . . . . . . . . 7

3.2 Generalized Morse sequences . . . . . . . . . . . . . . . . . . . 9 9

3.3 Bijective substitutions ....................... . . . 11

4 Sarnak's conjecture for finite group extensions 13

4.1 Lifting generic points for compact group extensions . . . . . . . . . . . . . 13

4.2 Criterion for the validity of Sarnak's conjecture for finite group extensions 14

4.3 Special case: 2-point extensions of odometers . . . . . . . . . . 15

5 Applications $\mathbf{1 6}$

5.1 Bijective substitutions . . . . . . . . . . . . . . . . . . . . 16

5.2 Regular Morse sequences and the Rudin-Shapiro case . . . . . . . . . . . 18

*Research supported by Narodowe Centrum Nauki grant UMO-2014/15/B/ST1/03736. 
6 Spectral approach and other methods

6.1 First remarks . . . . . . . . . . . . . . . . . . . . 19

6.2 Spectral approach . . . . . . . . . . . . . . . . . . 20

6.3 Applications . . . . . . . . . . . . . . . . . . . . . . . . . 20 21

6.4 Comparison with results of Veech $[47 \ldots \ldots \ldots \ldots \ldots$

\section{Introduction}

In 2010, Sarnak [45] formulated the following conjecture: for each zero entropy topological dynamical system $(T, X)$ ( $X$ is a compact metric space and $T$ is a homeomorphism of $X)$, each $f \in C(X)$ and $x \in X$, we have

$$
\frac{1}{N} \sum_{n \leqslant N} f\left(T^{n} x\right) \boldsymbol{\mu}(n) \rightarrow 0
$$

where $\boldsymbol{\mu}: \mathbb{N} \rightarrow \mathbb{C}$ is the Möbius function defined by $\boldsymbol{\mu}(1)=1, \boldsymbol{\mu}\left(p_{1} \cdot \ldots \cdot p_{k}\right)=(-1)^{k}$ for $k$ different prime numbers $p_{i}$, and $\boldsymbol{\mu}(n)=0$ in the remaining cases. The conjecture has already been proved in numerous cases, e.g. [1 15, 8, 11, 15, 16, 28, 35, 37, 38, 46, 47].

The aim of the present paper is to show that Sarnak's conjecture holds for some classes of dynamical systems of number theoretic origin. Namely, for all dynamical systems given by bijective substitutions (a subclass of substitutions of constant length) [43] and also for other related systems given by some automata (e.g. by the sequences of the Rudin-Shapiro type). Our approach is purely ergodic and the main tool is the theory of compact group extensions of rotations. Throughout, we deal with uniquely ergodic homeomorphisms, i.e. homeomorphisms possessing exactly one invariant measure (which has to be ergodic).

Given a bijective substitution $\theta$ over a finite alphabet $A$, we define its group cover substitution $\bar{\theta}$ over a subgroup $G$ of permutations of $A$, which hence carries an additional natural group structure. Since the dynamical system $(S, X(\theta))^{1}$ given by $\theta$ is a topological factor of the dynamical system $(S, X(\bar{\theta}))$ given by $\bar{\theta}$, it suffices to show that Sarnak's conjecture holds for $(S, X(\bar{\theta}))$. The group cover substitution $\bar{\theta}$ can be identified with a certain (generalized) Morse sequence $x$ and the associated dynamical system $(S, X(x))$ is a Morse system. This is where compact group extensions come into play - each Morse dynamical system is (measure-theoretically) isomorphic to a compact group extension $\left(T_{\psi}, X \times G\right)$ given by a so called Morse cocycle $\psi: X \rightarrow G$ over a rotation $T$, more precisely over an odometer $\left.(T, X)\right|^{2}$ The main difficulty is that such compact group extensions have been studied so far mostly from the measure-theoretic point of view [26, 33, 43] (the dynamical systems under considerations are uniquely ergodic). The underlying reason and, at the same time, the main obstacle for us is that Morse cocycles are in general not continuous. Thus, we cannot deal directly with such

\footnotetext{
${ }^{1}$ Here and in what follows, $S$ stands for the left shift on a closed $S$-invariant subset of the space of two-sided sequences, i.e. $(S, X(\theta))$ is an example of a subshift.

${ }^{2}$ This and other relations between the dynamical systems described in this paragraph are illustrated in Figure 1 .
} 
models - Sarnak's conjecture requires topological systems. In order to bypass this difficulty, more tools are used. The Morse dynamical system $(S, X(x))$ turns out to have a Toeplitz dynamical system $(S, X(\widehat{x}))$ as a topological factor, which, in turn, is an almost 1-1-extension of the odometer $(T, X)$. Moreover, the method of Toeplitz extensions [33] allows us to find a dynamical system topologically isomorphic to $(S, X(x))$, which also has a form of a compact group extension: $\left(S_{\varphi}, X(\widehat{x}) \times G\right)$ given by a continuous cocycle $\varphi: X(\widehat{x}) \rightarrow G$. If we denote the (natural) factoring map from $(S, X(\widehat{x}))$ to $(T, X)$ by $p$, we have the following relation between the two cocycles: $\varphi=\psi \circ p$. Our goal will be to prove that Sarnak's conjecture holds for $\left(S_{\varphi}, X(\widehat{x}) \times G\right)$.

The passage to group substitutions seems to be unavoidable for our method. Indeed, we require that the substitution subshift has a topological factor, determined by a Toeplitz sequence, which is an almost 1-1 extension of the maximal equicontinuous factor (the underlying odometer) and is measure-theoretically isomorphic to it. However, in [20], Section 4.4, it is proved that a bijective substitution need not have a symbolic factor which is measure-theoretically isomorphic to the maximal equicontinuous factor (this anwers a question raised by Baake). For example, this surprising property holds for the substitution $a \mapsto a a b a a, b \mapsto b c a b b$ and $c \mapsto c b c c c[20$.

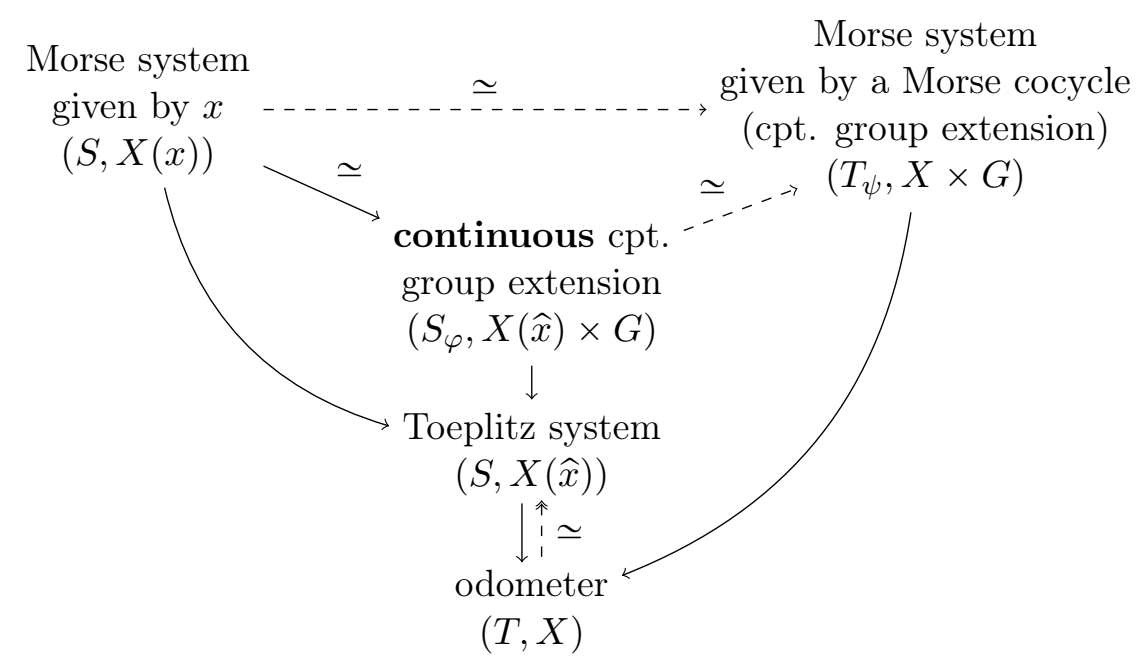

Figure 1: Morse and Toeplitz dynamical systems on a diagram. Plain and dashed lines denote topological and measure-theoretical maps, respectively (all depicted systems are uniquely ergodic).

The first tool we use to deal with the continuous compact group extensions is the Katai-Bourgain-Sarnak-Ziegler criterion:

Theorem 1.1 ([5, 24], see also [19]). Assume that $\left(a_{n}\right) \subset \mathbb{C}$ is bounded and suppose that

$$
\frac{1}{N} \sum_{n \leqslant N} a_{n r} \bar{a}_{n s} \rightarrow 0
$$


for all sufficiently large different prime numbers $r, s$. Then

$$
\frac{1}{N} \sum_{n \leqslant N} a_{n} \boldsymbol{\lambda}(n) \rightarrow 0
$$

for each multiplicativ $£^{3}$ function $\boldsymbol{\lambda}: \mathbb{N} \rightarrow \mathbb{C},|\boldsymbol{\lambda}| \leqslant 1$.

This criterion is applied to sequences of the form $a_{n}=f\left(T^{n} x\right), n \geqslant 1$, and, if satisfied, it yields a certain form of disjointness of different prime powers of the homeomorphism $T$; notice that ,sufficiently large" in Theorem 1.1 may depend on $f$ and $x$.

Our second tool is based on the method of lifting generic points in the Cartesian products of different prime powers $T^{r}$ and $T^{s}$ to almost 1-1-extensions. It has already appeared in [27], where the almost 1-1-extensions are chosen in such a way that the original cocycle considered on the extended space becomes continuous. Moreover, we will study ergodic joinings of $\left(T_{\psi}\right)^{r}$ and $\left(T_{\psi}\right)^{s}$ and show that in our case this set consists only of the relatively independent extensions of isomorphisms between $T^{r}$ and $T^{s}$ for different sufficiently large primes $r, s$. This will allow us to control generic points in the Cartesian product of the continuous compact group extension $\left(S_{\varphi}\right)^{r} \times\left(S_{\varphi}\right)^{s}$.

In Section 5, we give the main application of our method - we prove Sarnak's conjecture for the dynamical systems given by:

- all bijective substitutions (for a relationship of our result with a recent result of Drmota [10], see Remark 5.7),

- certain subclass of regular (generalized) Morse sequences,

- certain sequences of the Rudin-Shapiro type.

In Section 6, we compare our results concerning generalized Morse sequences and the Rudin-Shapiro type sequences with some earlier results in which (1) has been proved only for $f(y)=(-1)^{y[0]}$. Using spectral approach, we prove, in some cases, that the validity of (1) for such $f$ yields Sarnak's conjecture in its full form for the corresponding dynamical system. In particular, we show that Sarnak's conjecture holds for the dynamical systems given by Kakutani sequences [3, 15, 4 Sometimes, however, it seems that more than one function satisfying (1) is necessary for the validity of Sarnak's conjecture. E.g., this seems to be the case for the dynamical systems given by the Rudin-Shapiro type sequences. Here, (1) for $f$ has been been proved in [38, 46] by a purely combinatorial approach. The methods developed in [38] seem to be flexible enough to give (1) for finitely many functions described in Section 6.3, hence yields one more proof of Sarnak's conjecture for the corresponding dynamical system.

In Section 6.4, we compare our results with a recent work of Veech [47]. He provides a proof of Sarnak's conjecture for a class of dynamical systems given by some sequences

\footnotetext{
${ }^{3} \boldsymbol{\lambda}: \mathbb{N} \rightarrow \mathbb{C}$ is called multiplicative if $\boldsymbol{\lambda}(m \cdot n)=\boldsymbol{\lambda}(m) \cdot \boldsymbol{\lambda}(n)$ whenever $m, n$ are coprime. It is called aperiodic whenever $\frac{1}{N} \sum_{n \leqslant N} \boldsymbol{\lambda}(a n+b) \rightarrow 0$ for all $a, b \in \mathbb{N}$. The Möbius function $\boldsymbol{\mu}$ is multiplicative and aperiodic.

${ }^{4}$ This result does not seem to follow by Section 4 .
} 
over an arbitrary compact group. These sequences turn out to be generalizations of aforementioned Kakutani sequences. In particular, 47] gives an alternative proof of Sarnak's conjecture for Kakutani systems first proved in [3, 15].

\section{Basic tools}

\subsection{Spectral theory}

For an ergodic automorphism $T$ of a standard probability Borel space $(X, \mathcal{B}, \mu)$, we consider the associated Koopman operator on $L^{2}(X, \mathcal{B}, \mu)$ given by $U_{T}(f)=f \circ T$. Then there exist elements $f_{n} \in L^{2}(X, \mathcal{B}, \mu), n \geqslant 1$, such that

$$
L^{2}(X, \mathcal{B}, \mu)=\bigoplus_{n \geqslant 1} \mathbb{Z}\left(f_{n}\right) \text { and } \sigma_{f_{1}} \gg \sigma_{f_{2}} \gg \ldots,
$$

where $\mathbb{Z}(f)=\overline{\operatorname{span}}\left\{U_{T}^{n}(f): n \in \mathbb{Z}\right\}$ is the cyclic space generated by $f$ and $\sigma_{f}$ denotes the only finite positive Borel measure on $\mathbb{T}$ such that $\int_{X} f \circ T^{n} \cdot \bar{f} d \mu=\int_{\mathbb{T}} z^{n} d \sigma_{f}(z)$ for each $n \in \mathbb{Z}\left(\sigma_{f}\right.$ is called the spectral measure of $\left.f\right)$. The class of all measures equivalent to $\sigma_{f_{1}}$ in the above decomposition is called the maximal spectral type of $U_{T}$ and (2) is called a spectral decomposition. We say that the maximal spectral type is realized by $f \in L^{2}(X, \mathcal{B}, \mu)$ if $\sigma_{f}$ is equivalent to $\sigma_{f_{1}}$.

If $L^{2}(X, \mathcal{B}, \mu)=\mathbb{Z}\left(f_{1}\right) \oplus \cdots \oplus \mathbb{Z}\left(f_{k}\right)$ for some $f_{i} \in L^{2}(X, \mathcal{B}, \mu)$, we say that $U_{T}$ has multiplicity at most $k$. If no such $k \geqslant 1$ exists, the multiplicity of $U_{T}$ is infinite. If $k=1$, $U_{T}$ is said to have simple spectrum.

Recall that $T$ has discrete spectrum if the maximal spectral type of $U_{T}$ is purely discrete. Equivalently, $L^{2}(X, \mathcal{B}, \mu)$ is generated by the eigenfunctions of $U_{T}$. By the Halmos-von Neumann theorem, $T$ is, up to isomorphism, an ergodic rotation on a compact monothetic group. If, additionally, all eigenvalues of $U_{T}$ are roots of unity, $T$ is said to have rational discrete spectrum.

For more information on the spectral theory see, e.g., [42].

\section{$2.2 \quad$ Joinings}

Recall that if $T$ and $S$ are ergodic automorphisms on $(X, \mathcal{B}, \mu)$ and $(Y, \mathcal{C}, \nu)$, respectively, then by a joining between $T$ and $S$ we mean any $T \times S$-invariant measure $\kappa$ on $(X \times$ $Y, \mathcal{B} \otimes \mathcal{C}$ ) whose projections on $X$ and $Y$ are $\mu$ and $\nu$, respectively. We denote by $J(T, S)$ the set of joinings between $T$ and $S$ and by $J^{e}(T, S)$ the subset of ergodic joinings. Clearly, $\mu \otimes \nu \in J(T, S)$. If $T$ and $S$ are isomorphic, an isomorphism given by $R:(X, \mathcal{B}, \mu) \rightarrow(Y, \mathcal{C}, \nu)$, then the measure $\mu_{R}$ determined by $\mu_{R}(B \times C)=\mu\left(B \cap R^{-1} C\right)$, $B \in \mathcal{B}, C \in \mathcal{C}$, belongs to $J^{e}(T, S)$. It is concentrated on the graph of $R$ and is called a graph joining. When $T=S$, we speak about self-joinings of $T$ and each graph self-joining is given by some element from the centralizer $C(T)$ of $T !^{5}$

Suppose that $T$ and $S$ are isomorphic, where the isomorphism is given by $R: X \rightarrow Y$, and have extensions to $\bar{T}$ on $(\bar{X}, \overline{\mathcal{B}}, \bar{\mu})$ and $\bar{S}$ on $(\bar{Y}, \overline{\mathcal{C}}, \bar{\nu})$, respectively. The relatively

\footnotetext{
${ }^{5}$ The centralizer $C(T)$ consists of automorphisms of $(X, \mathcal{B}, \mu)$ commuting with $T$.
} 
independent extension of $\mu_{R}$ (to a joining of $\bar{T}$ and $\bar{S}$ ) is denoted by $\widetilde{\mu}_{R}$ and determined by

$$
\int_{\bar{X} \times \bar{Y}} F \otimes G d \widetilde{\mu}_{R}=\int_{X} \mathbb{E}(F \mid X) \cdot \mathbb{E}(G \mid Y) \circ R d \mu
$$

for $F \in L^{2}(\bar{X}, \bar{\mu}), G \in L^{2}(\bar{Y}, \bar{\nu})$.

\subsection{Compact group extensions}

Assume that $T$ is an ergodic automorphism of a standard Borel probability space $(X, \mathcal{B}, \mu)$. Let $G$ be a compact metric group with Haar measure $m_{G}$.

Definition 2.1. Any measurable map $\psi: X \rightarrow G$ is called a cocycle. The automorphism $T_{\psi}$ of $\left(X \times G, \mathcal{B} \otimes \mathcal{B}(G), \mu \otimes m_{G}\right)$ defined by

$$
T_{\psi}(x, g):=(T x, \psi(x) g)
$$

is called a $G$-extension of $T$ (it is an example of a compact group extension of $T$ ). We say that $\psi$ is ergodic if $T_{\psi}$ is ergodic.

Compact group extensions enjoy the following relative unique ergodicity property.

Lemma 2.1 ([13]). If $T_{\psi}$ is ergodic (i.e. if the product measure $\mu \otimes m_{G}$ is ergodic) then $\mu \otimes m_{G}$ is the only $T_{\psi}$-invariant measure projecting onto $\mu$.

Let $\tau_{g}$ be an automorphism of $\left(X \times G, \mu \otimes m_{G}\right)$ given by

$$
\tau_{g}\left(x, g^{\prime}\right)=\left(x, g^{\prime} \cdot g\right) \text { for each } g^{\prime} \in G .
$$

Then $T_{\psi} \circ \tau_{g}=\tau_{g} \circ T_{\psi}$, that is, $\tau_{g}$ is an element of the centralizer $C\left(T_{\psi}\right)$ of $T_{\psi}$.

Proposition 2.2 ([40]). Assume that $T$ is ergodic and $\psi: X \rightarrow G$ is ergodic as well. Assume additionally that $T$ has discrete spectrum. Then each $\widetilde{S} \in C\left(T_{\psi}\right)$ is a lift of some $S \in C(T)$. More precisely, $\widetilde{S}=S_{f, v}$, where $S_{f, v}(x, g)=(S x, f(x) v(g))$ for some $S \in$ $C(T)$, some measurable $f: X \rightarrow G$ and some continuous group automorphism $v: G \rightarrow G$. Moreover, if $\widetilde{S}$ and $\bar{S}$ are two lifts of $S \in C(T)$ then $\widetilde{S}=\bar{S} \circ \tau_{g_{0}}$ for some $g_{0} \in G$.

Definition 2.2. We will say that $T_{\psi}$ has $G$-trivial centralizer if

$$
C\left(T_{\psi}\right)=\left\{T_{\psi}^{k} \circ \tau_{g}: k \in \mathbb{Z}, g \in G\right\} .
$$

Definition 2.3. Let $H \subset G$ be a closed subgroup. The corresponding factorautomorphism $T_{\psi H}$ of $\left(X \times G / H, \mu \otimes m_{G / H}\right)$ given by

$$
T_{\psi H}(x, g H):=(T x, \psi(x) g H)
$$

is called a natural factor of $T_{\psi}$. It is called nontrivial if $H \neq G$, and it is called normal whenever $H$ is normal. 
Remark 2.3. Notice that a power of a group extension is clearly a group extension: $\left.\left(T_{\psi}\right)^{r}=T_{\psi^{(r)}}^{r}\right]^{6}$ and the passage to natural factors is "commutative": $\left(\left(T_{\psi}\right)^{r}\right)_{H}=\left(T_{\psi H}\right)^{r}$.

We need some facts about joinings of compact group extensions.

Theorem 2.4 (40]). Assume that $T$ is ergodic. Assume that $S \in C(T)$ and let $\psi_{i}$ : $X \rightarrow G$ be an ergodic cocycle, $i=1,2$. Assume that $\kappa \in J^{e}\left(T_{\psi_{1}}, T_{\psi_{2}}\right)$ and projects on the graph self-joining $\mu_{S}$ of $T$. Then there are two closed normal subgroups $H_{1}, H_{2} \subset G$ and an isomorphism $\bar{S}$ (a lift of $S$ ) between the two normal natural factors $T_{\psi_{1} H_{1}}$ and $T_{\psi_{2} H_{2}}$ such that

$$
\kappa=\left(\widetilde{m_{G / H_{1}}}\right)_{\bar{S}},
$$

i.e. $\kappa$ is the relatively independent extension of the graph joining $\left(m_{G / H_{1}}\right)_{\bar{S}} \in$ $J^{e}\left(T_{\psi_{1} H_{1}}, T_{\psi_{2} H_{2}}\right)$ given by the isomorphism $\bar{S}$.

Remark 2.5. Suppose that $T$ has rational discrete spectrum and $\left(T_{\psi}\right)^{r}$ and $\left(T_{\psi}\right)^{s}$ are ergodic. Then $T^{r}$ and $T^{s}$ are isomorphic and the only ergodic joinings between them are the graph joinings. By Theorem 2.4 if there is no isomorphism between nontrivial normal natural factors of $\left(T_{\psi}\right)^{r}$ and $\left(T_{\psi}\right)^{s}$, then there are no ergodic joinings between $\left(T_{\psi}\right)^{r}$ and $\left(T_{\psi}\right)^{s}$, except for the "most independent" ones: the relatively independent extensions of isomorphisms between $T^{r}$ and $T^{s}$. Notice also that if such a relative product is ergodic then automatically, by Lemma 2.1, it is the only invariant measure on $X \times G \times X \times G$ projecting on the graph of the isomorphism.

\subsection{Generic points}

Let $T$ be a homeomorphism of a compact metric space $X$. Let $\mu$ be a $T$-invariant Borel probability measure on $X$.

Definition 2.4. We say that $x \in X$ is generic for $\mu$ if $\frac{1}{N} \sum_{n \leqslant N} \delta_{T^{n} x} \rightarrow \mu$ weakly. If the convergence to $\mu$ takes place only along a subsequence $\left(N_{k}\right)$ then $x$ is called quasi-generic for $\mu$.

Remark 2.6. Notice that, by the compactness of $X$, the space of probability measures on $X$ is also compact, hence each point is quasi-generic for some $T$-invariant measure.

\section{$3 \quad$ Basic objects}

\subsection{Odometers, Morse cocycles and Toeplitz extensions}

Odometers Assume that $\left(n_{t}\right)_{t \geqslant 0}$ satisfies $n_{0}=1$ and $n_{t} \mid n_{t+1}$ with $\lambda_{t}:=n_{t+1} / n_{t} \geqslant 2$ for $t \geqslant 0$. Consider $X:=\prod_{t \geqslant 0} \mathbb{Z} / \lambda_{t} \mathbb{Z}$ with the product topology and the group law given

\footnotetext{
${ }^{6} \psi^{(r)}(x):=\psi(x) \psi(T x) \ldots \psi\left(T^{r-1} x\right)$ for $r \geqslant 0$ and extends to $r \in \mathbb{Z}$ so that the cocycle identity $\psi^{(m+n)}(x)=\psi^{(m)}(x) \psi^{(n)}\left(T^{m} x\right)$ holds for every $m, n \in \mathbb{Z}$.
} 
by addition mod $\lambda_{t}$, with carrying the remainder to the right. This makes $X$ a compact metric Abelian group. We define the translation $T$ by $(1,0,0, \ldots)$ :

$$
T\left(x_{0}, x_{1}, x_{2}, \ldots\right)=\left(x_{0}+1, x_{1}, x_{2}, \ldots\right)
$$

to obtain $\left(X, \mathcal{B}, m_{X}, T\right)$ - an ergodic rotation.

Definition 3.1. $T$ defined above is called an odometer.

Remark 3.1. Odometer $T$ defined above has rational discrete spectrum given by the $n_{t^{-}}$ roots of unity, $t \geqslant 0$. For each $t \geqslant 0$, there is a Rokhlin tower $\mathcal{D}^{t}:=\left\{D_{0}^{t}, D_{1}^{t}, \ldots, D_{n_{t}-1}^{t}\right\}$, i.e. a partition of $X$ for which $T^{i} D_{0}^{t}=D_{i \bmod n_{t}}^{t}$ for each $i \geqslant 0$ (by ergodicity, such a tower is unique up to cyclic permutation of the levels). Indeed, $D_{0}^{0}=X$ and we set

$$
D_{0}^{t}:=\left\{x \in X: x_{0}=\ldots=x_{t-1}=0\right\}, t \geqslant 1 .
$$

Clearly, the partition $\mathcal{D}^{t+1}$ is finer that $\mathcal{D}^{t}$ and the sequence of such partitions tends to the partition into points.

Remark 3.2 (cf. Remark 2.5). Notice that for each $r \geqslant 1$,

$$
T^{r} \text { is isomorphic to } T \text { whenever } T^{r} \text { is ergodic. }
$$

Indeed, $T^{r}$ has the same spectrum as $T$. To see the isomorphism more directly, notice that $\operatorname{gcd}\left(r, n_{t}\right)=1$ and $T^{r}$ permutes the levels of $\mathcal{D}^{t}$ - this extends to an isomorphism map between $T$ and $T^{r}$.

Remark 3.3 (cf. Remark 2.5). Since $T$ has discrete spectrum, its only ergodic joinings are graph measures $\left(m_{X}\right)_{W}$, where $W \in C(T)$ is another rotation on $X$ [14]. It easily follows that

$$
\begin{aligned}
& \text { each point }(x, y) \in X \times X \text { is generic for an ergodic self-joining of the } \\
& \text { form }\left(m_{X}\right)_{W} \text {. }
\end{aligned}
$$

Indeed, define $W$ as the translation by $x-y$.

Morse cocycles Assume that $G$ is a compact metric group and $(T, X)$ is an odometer.

Definition 3.2 ([17, 33]). We say that $\psi: X \rightarrow G$ is a Morse cocycle if $\psi$ is constant on each $D_{i}^{t}, t \geqslant 0, i=0,1, \ldots, n_{t}-2\left(\left.\psi\right|_{D_{i}^{t}}\right.$ may depend on $\left.i\right)$.

Remark 3.4. To see what are the values of a Morse cocycle $\psi$ on $D_{n_{t}-1}^{t}$, we first pass to the levels $D_{j n_{t}-1}^{t+1}$ for $j=1, \ldots, \lambda_{t+1}-1$, and read the values $\left.\psi\right|_{D_{j n_{t}-1}^{t+1}}$. To read the values on $D_{n_{t+1}-1}^{t+1}\left(n_{t+1}=\lambda_{t+1} n_{t}\right)$, we pass to $\mathcal{D}^{t+2}$ etc. It is clear that $\psi$ defined in this way is continuous everywhere (as the levels of the towers are clopen sets) except perhaps one point (given by the intersection of the top levels of all towers). Notice also that whenever $G$ is finite then a Morse cocycle cannot be continuous unless it is constant on each level of the tower $\mathcal{D}^{t_{0}}$ for some $t_{0}$. In this case, $T_{\psi}$, if ergodic, is a direct product of $T$ with a rotation on $G$. In particular, Sarnak's conjecture holds for $T_{\psi}$.

Remark 3.5. The class of group extensions given by Morse cocycles is (up to measuretheoretic isomorphism) the same as the class of dynamical systems generated by generalized Morse sequences, see [17, 26, 33, 44], which we consider in the next section. 
Toeplitz extensions Morse cocycles yield extensions of odometers which are special cases of so called Toeplitz extensions studied in [33] (cf. earlier [48, 49] and the last section). Toeplitz extensions are also given by cocycles over odometers but in the definition of such cocycles we are letting more than one level have non-constant values, as in the example below 7

Example 3.1. Let $\lambda_{t}:=2$ for each $t \geqslant 0$ and $G:=\mathbb{Z} / 2 \mathbb{Z}$. We define $\psi: X \rightarrow \mathbb{Z} / 2 \mathbb{Z}$ so that at stage $t$ it is defined on each $D_{i}^{t}$, except for $i=2^{t-1}-1$ and $i=2^{t}-1$. Then, when we pass to $\mathcal{D}^{t+1}$, on the levels $D_{2^{t-1}-1}^{t+1}$ and $D_{2^{t}+2^{t-1}-1}^{t+1}(\psi$ must be defined here at this stage of the construction), we set the values 0 and 1 (or 1 and 0 ), respectively.

The class of Toeplitz extensions of the dyadic odometer described in Example 3.1 was considered in [33, 36]. The dynamical systems corresponding to the Rudin-Shapiro type sequences (see Section III.2 in [33]) are in this class.

\subsection{Generalized Morse sequences}

Let $G$ be a compact metric group with the unit $e$.

Definition 3.3. Let $b^{t} \in G^{\lambda_{t}}$ be a block over $G$ of length $\left|b^{t}\right|=\lambda_{t} \geqslant 2$ and $b^{t}[0]=e$, $t \geqslant 0$. The associated (generalized) Morse sequence is defined by

$$
x:=b^{0} \times b^{1} \times \ldots,
$$

where $B \times C=(B \circ c[0])(B \circ c[1]) \ldots(B \circ c[|C|-1])$ and $B \circ g:=\left(b_{0} g, \ldots, b_{|B|-1} g\right)$ for $B, C$ blocks over $G$ and $g \in G$. By $(S, X(x))$ we denote the subshift corresponding to $x$ $\left(X(x) \subset G^{\mathbb{Z}}\right)$.

Example 3.2. Generalized Morse sequences for $G=\mathbb{Z} / 2 \mathbb{Z}$ were first studied in [26]. If $b^{t} \in\{00,01\}, t \geqslant 0$, we speak about Kakutani sequences [29].

Definition $3.4([22])$. We say that $u \in G^{\mathbb{N}}$ is a Toeplitz sequence whenever for each $n \in \mathbb{N}$ there exists $k_{n} \geqslant 1$ such that $u$ is constant on the arithmetic progression $n+k_{n} \mathbb{N} 8$

Lemma 3.6 (cf. Figure 1). Let $x=b^{0} \times b^{1} \times \ldots$ be a Morse sequence. The map

$$
y \mapsto \widehat{y}, \widehat{y}[n]:=y[n+1] y[n]^{-1}
$$

yields an equivariant map between $(S, X(x))$ and $(S, X(\widehat{x}))$. Moreover, $\widehat{x}$ is a Toeplitz sequence.

\footnotetext{
${ }^{7}$ It is however required that the numbers of levels of $\mathcal{D}^{t}$ on which the cocycle is non-constant divided by $n_{t}$ goes to zero. A reason for that is that we want to obtain a regular Toeplitz sequence which is behind such a construction, see 33 for more details.

${ }^{8}$ For the theory of dynamical systems given by Toeplitz sequences, see e.g. [7].
} 
Proof. The first part is obvious. For the second, notice that for each $t \geqslant 1$, we have

$$
x=c_{t} \times z_{t}, \text { where } c_{t}=b^{0} \times \ldots \times b^{t-1}, z_{t}=b^{t} \times b^{t+1} \times \ldots,
$$

whence $x$ is a concatenation of blocks of the form $c_{t} \circ g$. Moreover,

$$
c_{t}[n+1] c_{t}[n]^{-1}=\left(c_{t} \circ g\right)[n+1]\left(c_{t} \circ g\right)[n]^{-1} \text { for } n=0, \ldots,\left|c_{t}\right|-2 .
$$

It follows that

$$
\widehat{x}=\widehat{c}_{t} * \widehat{c}_{t} * \widehat{c}_{t} * \ldots,
$$

where "*" stands for the unfilled place of $\widehat{x}$ at the stage $t \geqslant 1$.

Remark 3.7. Toeplitz sequence $\widehat{x}$ from (7) is regular in the sense of [22]. Hence $(S, X(\widehat{x}))$ is uniquely ergodic [7].

Lemma 3.8 (cf. Figure 1). $(S, X(x))$ is topologically isomorphic to $\left(S_{\varphi}, X(\widehat{x}) \times G\right)$, where $\varphi: X(\widehat{x}) \rightarrow G$ is the continuous cocycle given by

$$
\varphi(z)=z[0], \text { for each } z \in X(\widehat{x}) .
$$

Proof. The topological isomorphism is given by the (equivariant) map

$$
y \mapsto(\widehat{y}, y[0]) .
$$

Indeed, this map is continuous as $\varphi$ is continuous, it is onto since $\widehat{y}=\widehat{y \circ g}$, and finally it is $1-1$ since $y[n+1]$ is determined by $y[n]$ and $\widehat{y}[n]$.

Remark 3.9 (cf. Figure 1). Let $x$ be the Morse sequence given by (5) with $n_{t}:=\left|c_{t}\right|=$ $\left|b^{0} \times \cdots \times b^{t-1}\right|, t \geqslant 1$. Then the Toeplitz system $(S, X(\widehat{x}))$ has the $\left\{n_{t}\right\}$-odometer $(T, X)$ as its topological factor. Moreover, the Morse dynamical system $(S, X(x))$ is given by a Morse cocycle $\psi$ over $T$. The values $\left(\left.\psi\right|_{D_{0}^{t}}, \ldots,\left.\psi\right|_{D_{n_{t}-2}^{t}}\right)$ are determined by $\widehat{c}_{t}$ :

$$
\left.\psi\right|_{D_{i}^{t}}=\widehat{c}_{t}[i] \text { for } 0 \leqslant i \leqslant n_{t}-2, t \geqslant 1 \text {. }
$$

It follows that

$$
b^{0}[0]=e, b^{0}[i]=\prod_{j=0}^{i-1} \widehat{c}_{1}[j], 1 \leqslant i \leqslant \lambda_{0}-1
$$

and then, inductively $\left(c_{t}=b^{0} \times \ldots \times b^{t-1}\right)$,

$$
b^{t+1}[0]=e, b^{t+1}[i]=\prod_{j=i}^{1}\left(\widehat{c}_{t+1}\left[j n_{t}-1\right] c_{t}\left[n_{t}-1\right]\right), 1 \leqslant i \leqslant \lambda_{t+1}-1
$$

(for more details see, e.g. [9, 33]). However, the Morse cocycle is not continuous. The passage to the Toeplitz dynamical system from Lemma 3.8 allows us to get its continued version.

Lemma 3.10. The normal natural factors of Morse dynamical systems over $G$ are Morse dynamical systems over $G / H$.

Proof. The assertion follows immediately from the equality $B \times C \bmod H=(B \bmod H) \times$ $(C \bmod H)$ and Remark 3.9 . 


\subsection{Bijective substitutions}

Fix a finite alphabet $A$ with $|A|=r \geqslant 2$.

Definition 3.5. A map $\theta: A \rightarrow A^{\lambda}(\lambda \geqslant 1)$ is called a substitution on $A$ of constant length $\lambda$ (in what follows, simply a substitution) if there exists $n \geqslant 1$ such that for each $a, a^{\prime} \in A$ there exists $k$ satisfying $\theta^{n}(a)[k]=a^{\prime}$. We extend $\theta$ first to a map on blocks over $A$, then to a map $\theta: A^{\mathbb{N}} \rightarrow A^{\mathbb{N}}$. We will assume that $\theta\left(a_{0}\right)[0]=a_{0}$. By iterating $\theta$ at $a_{0}$, we obtain a fixed point for the map $\theta: A^{\mathbb{N}} \rightarrow A^{\mathbb{N}}$ and we denote by $(S, X(\theta))$ the corresponding subshift of $A^{\mathbb{Z}}$.

Remark 3.11 (see Chapter 5 in [43]). For each substitution $\theta,(S, X(\theta))$ is strictly ergodic.

Remark 3.12. Let $\theta: A \rightarrow A^{\lambda}$ be a substitution such that $(S, X(\theta))$ is aperiodic. Recall that then for each $y \in X(\theta)$ there is a unique sequence $\left(i_{t}(y)\right)_{t \geqslant 1} \subset \mathbb{Z}$ (t-skeleton) with $i_{t}(y) \in\left[-\lambda^{t}+1,0\right]$ such that $y\left[i_{t}+k \lambda^{t}, i_{t}+(k+1) \lambda^{t}-1\right]=\theta^{t}\left(a_{k, t}\right)$ for each $k \in \mathbb{Z}$ and some letters $a_{k, t} \in A$. This allows us to define the corresponding towers of height $\lambda^{t}$ by setting the base of the $t$-tower

$$
D_{0}^{t}:=\left\{y \in X(\theta): i_{t}(y)=0\right\}
$$

to obtain $\bigcup_{i=0}^{\lambda^{t}-1} S^{i} D_{0}^{t}=X(\theta)$.

Definition 3.6 ([41]). We say that substitution $\theta$ is recognizable if there exists a constant $M>0$ such that if $y \in X(\theta), t \geqslant 1$ and $i \in\left[-\lambda^{t}+1,0\right]$ satisfy

$$
y\left[i, i+M \lambda^{t}-1\right]=\theta^{t}\left(b_{1}\right) \ldots \theta^{t}\left(b_{M}\right)
$$

for some $b_{1}, \ldots, b_{M} \in A$ then $i=i_{t}(y)$. We say that $M$ is a constant of recognizability.

Remark $3.13(41,43])$. Each substitution $\theta$ such that $(S, X(\theta))$ is aperiodic, is recognizable. In what follows, we will tacitly assume that we deal with recognizable substitutions.

Remark 3.14. Suppose that $\theta$ is recognizable. It follows that each function $\mathbb{1}_{D_{0}^{t}}$ depends on not more than $M \lambda^{t}$ coordinates.

Definition 3.7 ([4]). Substitution $\theta$ is called bijective if

$$
\bar{d}\left(\theta(a), \theta\left(a^{\prime}\right)\right):=\frac{\left|\left\{0 \leqslant k \leqslant \lambda-1: \theta(a)[k] \neq \theta\left(a^{\prime}\right)[k]\right\}\right|}{\lambda}=1, \text { whenever } a \neq a^{\prime}
$$

or, equivalently, the maps $\sigma_{i}(a):=\theta(a)[i]$ are bijections of $A, i=0, \ldots, \lambda-1$.

Remark 3.15. We can assume (wlog) that $\sigma_{0}=I d$ by considering, if necessary, its power.

Definition 3.8. Let $G$ be a finite group with the unit $e$. A substitution $\theta: G \rightarrow G^{\lambda}$ is called a group substitution whenever

$$
\theta(g)=\theta(e) \circ g \text { for each } g \in G .
$$


Remark 3.16. Each group substitution is bijective. Moreover, each group substitution can be identified with the Morse sequence $\theta(e) \times \theta(e) \times \ldots$

Lemma 3.17 (cf. Lemma 3.10 and Remark 3.16). The normal natural factors of $d y$ namical systems given by group substitutions are determined by group substitutions.

Proof. Consider the group substitution given by $e \mapsto B$ (i.e. $g \mapsto B \circ g$ ). Then

$$
g H \mapsto B \circ g \bmod H
$$

yields a bijective substitution as in each column of the matrix corresponding to the group substitution we see all elements of $G$; in particular, by taking them $\bmod H$, we see all elements of $G / H$.

Denote by $\mathscr{S}_{r}$ the group of permutations of $A$. Define $\widetilde{\theta}: \mathscr{S}_{r} \rightarrow \mathscr{S}_{r}^{\lambda}$ by setting

$$
\tilde{\theta}(\tau)=\left(\sigma_{0} \circ \tau, \sigma_{1} \circ \tau, \ldots, \sigma_{\lambda-1} \circ \tau\right)=\tilde{\theta}(I d) \circ \tau
$$

for each $\tau \in \mathscr{S}_{r}$. Let $G \subset \mathscr{S}_{r}$ be the subgroup generated by $\sigma_{0}, \sigma_{1}, \ldots, \sigma_{\lambda-1}$ and define

$$
\bar{\theta}(\tau):=\widetilde{\theta}(\tau) \text { for } \tau \in G .
$$

Definition 3.9 (cf. Lemma 3.19 below). We call $\bar{\theta}$ the group cover substitution of $\theta$.

Lemma 3.18. $\bar{\theta}$ is a (bijective) substitution.

Proof. Notice that if $\bar{\theta}^{n}\left(\sigma_{0}\right)[j]=\tau$ then, in $\bar{\theta}^{n+1}\left(\sigma_{0}\right)$, we can find the block $\left(\sigma_{0} \circ\right.$ $\left.\tau, \ldots, \sigma_{\lambda-1} \circ \tau\right)$. Since all elements in $G$ are of finite order $\left(\sigma_{i}^{-1}=\sigma_{i}^{r !-1}\right)$, it follows by induction that, for some $n$, we will see all symbols from $G$ on $\bar{\theta}^{n}\left(\sigma_{0}\right)$.

Lemma 3.19. $(S, X(\theta))$ is a topological factor of its group cover substitution $(S, X(\bar{\theta}))$.

Proof. We define an equivalence relation on $G$ by setting $\tau \equiv \tau^{\prime}$ if $\tau(0)=\tau^{\prime}(0){ }^{9}$ For $y \in X(\bar{\theta})$, set

$$
F(y)[n]:=(y[n])(0) .
$$

Notice that the image of $F$ equals $X(\theta), F$ is equivariant and takes the same values on the equivalence classes of $\equiv$. Finally, notice that $\{\tau(0): \tau \in G\}=A$ since $\theta$ is a substitution, whence $G$ acts transitively on $A$.

Remark 3.20 (cf. Remark 3.16). The group cover substitution $\bar{\theta}$ can be identified with the Morse sequence $B \times B \times \ldots$ (over $G$ ), where $B=\left(\sigma_{0}, \sigma_{1}, \ldots, \sigma_{\lambda-1}\right)$.

Remark 3.21. Notice that, in order to prove Sarnak's conjecture for a bijective substitution $\theta$, it suffices to prove it for $\left(S_{\varphi}, X(\widehat{x}) \times G\right)$, where $x=B \times B \times \ldots$ as $(S, X(\theta))$ is its topological factor (see Lemma 3.19 and Remark 3.20 . Notice also that we do not claim that for $(S, X(\theta))$ the Toeplitz dynamical system $(S, X(\widehat{x}))$ is its topological factor (even though the odometer is its topological factor). In fact, there is a counterexample to such a claim due to Herning [20].

\footnotetext{
${ }^{9}$ This relation is called $\theta$-consistent, see [23, 39].
} 


\section{Sarnak's conjecture for finite group extensions}

\subsection{Lifting generic points for compact group extensions}

We now recall a basic result on lifting generic points from [27]. Assume that $\bar{T}_{i}\left(T_{i}\right)$ is a uniquely ergodic homeomorphism, with a unique invariant measure $\bar{\mu}_{i}\left(\mu_{i}\right)$, of a compact metric space $\bar{X}_{i}\left(X_{i}\right), i=1,2$. Assume, moreover, that $\pi_{i}: \bar{X}_{i} \rightarrow X_{i}$ is continuous and yields $\left(T_{i}, X_{i}\right)$ a topological factor of $\left(\bar{T}_{i}, \bar{X}_{i}\right)$.

Proposition 4.1 ([27]). Assume that $\left(\bar{T}_{i}, \bar{X}_{i}, \bar{\mu}_{i}\right)$ and $\left(T_{i}, X_{i}, \mu_{i}\right)$ are measuretheoretically isomorphic. Assume, moreover that $\left(T_{i}, X_{i}, \mu_{i}\right)$ is measure-theoretically coalescen ${ }^{10}$ for $i=1,2$. Assume that $\left(x_{1}, x_{2}\right) \in X_{1} \times X_{2}$ is generic for an ergodic $T_{1} \times T_{2}$ invariant measure $\rho$. Then there exists a unique $\bar{T}_{1} \times \bar{T}_{2}$-invariant measure $\bar{\rho}$, such that each pair $\left(\bar{x}_{1}, \bar{x}_{2}\right) \in\left(\pi_{1} \times \pi_{2}\right)^{-1}\left(x_{1}, x_{2}\right)$ is generic for $\bar{\rho}$. Moreover, the system $\left(\bar{T}_{1} \times \bar{T}_{2}, \bar{\rho}\right)$ is isomorphic to $\left(T_{1} \times T_{2}, \rho\right)$.

Let $T$ be an odometer acting on $\left(X, \mathcal{B}, m_{X}\right)$ and let $\bar{T}$ be a uniquely ergodic homeomorphism of $\bar{X}$ (with the unique invariant measure $\overline{m_{X}}$ ) such that $\pi: \bar{X} \rightarrow X$ is a topological factor map, and $\left(T, m_{X}\right)$ and $\left(\bar{T}, \bar{m}_{X}\right)$ are measure-theoretically isomorphic (then $\pi$ is a.e. 1-1 as transformations with discrete spectrum are coalescent). Assume that $\psi: X \rightarrow G$ is ergodic and such that the cocycle $\bar{\psi}: \bar{X} \rightarrow G$ given by

$$
\bar{\psi}(\bar{x}):=\psi(\pi(\bar{x})) \text { is continuous }
$$

(it is automatically ergodic as $\left(T_{\psi}, X \times G, m_{X} \otimes m_{G}\right)$ and $\left(\bar{T} \bar{\psi}, \bar{X} \times G, \bar{m}_{X} \otimes m_{G}\right)$ are isomorphic). Assume that $r \neq s$ are such that $\left(T_{\psi}\right)^{r}$ and $\left(T_{\psi}\right)^{s}$ are ergodic, hence $T^{r}$ and $T^{s}$ are isomorphic (and they are isomorphic to $T$ ).

Proposition 4.2. Assume that the only ergodic joinings between $\left(T_{\psi}\right)^{r}$ and $\left(T_{\psi}\right)^{s}$ are the relatively independent extensions over the graphs of isomorphisms between $T^{r}$ and $T^{s}$. Let $\bar{x} \in \bar{X}$ and let $\rho=\left(m_{X}\right)_{R}$ be the (ergodic) graph joining for which the point $(\pi(\bar{x}), \pi(\bar{x}))$ is generic. Then for each $g \in G$, the point $((\bar{x}, g),(\bar{x}, g))$ is generic for the $\tilde{\bar{\rho}}$, where $\bar{\rho}$ comes from Proposition 4.1 ( $\sim$ stands for the relatively independent extension). Moreover,

$$
\left(\left(\bar{T}_{\bar{\psi}}\right)^{r} \times\left(\bar{T}_{\bar{\psi}}\right)^{s}, \tilde{\bar{\rho}}\right) \text { and }\left(\left(T_{\psi}\right)^{r} \times\left(T_{\psi}\right)^{s}, \widetilde{\left(m_{X}\right)_{R}}\right) \text { are isomorphic. }
$$

Proof. The point $((\bar{x}, g),(\bar{x}, g))$ is quasi-generic for a $\left(\bar{T}_{\bar{\psi}}\right)^{r} \times\left(\bar{T}_{\bar{\psi}}\right)^{s}$-invariant measure $\kappa$. By Proposition 4.1. $(\bar{x}, \bar{x})$ is generic for $\bar{\rho}$. Therefore, the projection of $\kappa$ on $\bar{X} \times \bar{X}$ is equal to $\bar{\rho}$. Using Lemma 2.1 (applied to $\bar{T}^{r} \times \bar{T}^{s}, \bar{\psi}^{(r)} \times \bar{\psi}^{(s)}$ and $\bar{\rho}$ ), to conclude, we only need to prove that $\left(\left(\bar{T}_{\bar{\psi}}\right)^{r} \times\left(\bar{T}_{\bar{\psi}}\right)^{s}, \tilde{\bar{\rho}}\right)$ is ergodic. Notice that $(11)$ is obvious since $\rho=\left(m_{X}\right)_{R}$ and $\bar{\rho}$ yield isomorphic systems. This gives immediately that $\left(\left(\bar{T}_{\bar{\psi}}\right)^{r} \times\left(\bar{T}_{\bar{\psi}}\right)^{s}, \tilde{\bar{\rho}}\right)$ is ergodic, whence $\kappa=\widetilde{\bar{\rho}}$.

\footnotetext{
${ }^{10}$ An automorphism $T$ of $(X, \mathcal{B}, \mu)$ is called coalescent [18] if each endomorphism commuting with $T$ is invertible.
} 


\subsection{Criterion for the validity of Sarnak's conjecture for finite group extensions}

In this section, we assume that $(T, X, \mathcal{B}, \mu)$ is an ergodic transformation with discrete spectrum and $\psi: X \rightarrow G$ is an ergodic cocycle with values in a finite group $G$.

Lemma 4.3. Let $m=|G|$. Assume that $r \geqslant 2$ is an integer such that $\left(T_{\psi}\right)^{r}$ is ergodic and $\operatorname{gcd}(r, m)=1$. Then $C\left(T_{\psi}\right)=C\left(\left(T_{\psi}\right)^{r}\right)$.

Proof. Assume that $\widetilde{S} \in C\left(\left(T_{\psi}\right)^{r}\right)$. Since $T_{\psi} \in C\left(\left(T_{\psi}\right)^{r}\right)$, we have $\left(T_{\psi}\right)^{-1} \circ \widetilde{S} \circ T_{\psi} \in$ $C\left(\left(T_{\psi}\right)^{r}\right)$. Since $\left(T_{\psi}\right)^{-1} \circ \widetilde{S} \circ T_{\psi} \in C\left(\left(T_{\psi}\right)^{r}\right)$ is a lift of $S$ and $\left(T_{\psi}\right)^{r}$ is ergodic, it follows by Proposition 2.2 that

$$
\left(T_{\psi}\right)^{-1} \circ \widetilde{S} \circ T_{\psi}=\widetilde{S} \circ \tau_{g} \text { for some } g \in G .
$$

Therefore

$$
\left(T_{\psi}\right)^{-2} \circ \widetilde{S} \circ\left(T_{\psi}\right)^{2}=\left(T_{\psi}\right)^{-1} \circ \widetilde{S} \circ \tau_{g} \circ T_{\psi}=\left(T_{\psi}\right)^{-1} \circ \widetilde{S} \circ T_{\psi} \circ \tau_{g}=\widetilde{S} \circ \tau_{g^{2}}
$$

and, in a similar way, $\left(T_{\psi}\right)^{-m} \circ \widetilde{S} \circ\left(T_{\psi}\right)^{m}=\widetilde{S} \circ \tau_{g^{m}}=\widetilde{S}$, i.e. $\widetilde{S} \in C\left(\left(T_{\psi}\right)^{m}\right)$. Let $a, b \in \mathbb{Z}$ be such that $a m+b r=1$. We conclude that $\widetilde{S}$ commutes with $\left(T_{\psi}\right)^{a m+b r}=T_{\psi}$ which completes the proof.

Proposition 4.4. Assume that $T_{\psi}$ has continuous spectrum on the orthocomplement of $L^{2}(X, \mathcal{B}, \mu) \otimes \mathbb{1}_{G}$. Suppose that $r \geqslant 2$ is such that $T^{r}$ is ergodic and $\operatorname{gcd}(m, r)=1$. Then $C\left(T_{\psi}\right)=C\left(\left(T_{\psi}\right)^{r}\right)$.

Proof. Since, by assumptions, $\left(T_{\psi}\right)^{r}$ is ergodic, the assertion follows from Lemma 4.3 .

We can now formulate a general criterion concerning the validity of Sarnak's conjecture for continuous finite group extensions.

Proposition 4.5. Let $\bar{T}$ be a uniquely ergodic homeomorphism which is a continuous extension of an odometer $T$, measure-theoretically isomorphic to T. Assume that its (rational discrete) spectrum is determined by finitely many prime numbers. Assume that $\psi: X \rightarrow G$ is a cocycle with $G$ finite, $(10)$ is satisfied, and $T_{\psi}$ has continuous spectrum in the orthocomplement of $L^{2}\left(X, \mathcal{B}, m_{X}\right) \otimes \mathbb{1}_{G}$. Assume moreover that the centralizers for all normal natural factors $T_{\psi H}$ of $T_{\psi}$ are $G / H$-trivial whenever $H \neq G$. Then, for each $f \in C(X)$ and $j \in C(G)$ of zero mean, (1) is satisfied for $\bar{T}_{\bar{\psi}}$ and $(f \circ \pi) \otimes j \in C(\bar{X} \times G)$ at each point.

Proof. Fix $r, s$ two different prime numbers sufficiently large (so that $\left(T_{\psi}\right)^{r}$ and $\left(T_{\psi}\right)^{s}$ are ergodic). Notice that $T^{r}$ is then isomorphic to $T^{s}$. Following Theorem 2.4 (applied to $T^{r}$ isomorphic to $T^{s}$, both isomorphic to $T$ ) and Remark 2.5, we first will prove that if $H_{1}, H_{2}$ are proper normal subgroups of $G$ then $\left(T_{\psi H_{1}}\right)^{r}$ is not isomorphic $\left(T_{\psi H_{2}}\right)^{s}$. For this aim, it is enough to notice is that $\left(T_{\psi H_{2}}\right)^{s}$ cannot have an $r$-th root. Indeed, using 
the fact that the centralizer of $\left(T_{\psi H_{2}}\right)^{s}$ is $G / H_{2}$-trivial and Proposition 4.4 , if $\left(T_{\psi H_{2}}\right)^{s}$ an $r$-th root then

$$
\left(T_{\psi H_{2}}\right)^{s}=\left(\left(T_{\psi H_{2}}\right)^{k} \circ \tau_{g H_{2}}\right)^{r}=\left(T_{\psi H_{2}}\right)^{k r} \circ \tau_{g^{r} H_{2}} .
$$

It follows that $\tau_{g^{r} H_{2}}=\left(T_{\psi H_{2}}\right)^{s-k r}$, which is an absurd as $s, r$ are prime $(s \neq k r)$ and $T_{\psi}$ is aperiodic.

Take any $(\bar{x}, g)$. By the first part of the proof and Proposition 4.2 , we obtain

$$
\frac{1}{N} \sum_{n \leqslant N} \delta_{\left(\bar{T}_{\bar{\psi}}\right)^{r n} \times\left(\bar{T}_{\bar{\psi}}\right)^{s n}((\bar{x}, g),(\bar{x}, g))} \rightarrow \widetilde{\bar{\rho}}
$$

Therefore

$$
\begin{array}{r}
\frac{1}{N} \sum_{n \leqslant N}((f \circ \pi) \otimes j)\left(\bar{T}_{\bar{\psi}}\right)^{r n} \times\left(\bar{T}_{\bar{\psi}}\right)^{s n}((\bar{x}, g),(\bar{x}, g)) \rightarrow \int((f \circ \pi) \otimes j) \cdot \overline{((f \circ \pi) \otimes j)} d \tilde{\bar{\rho}} \\
=\int(f \otimes j) \cdot \overline{(f \otimes j)} d \widetilde{\mu_{R}}=\int_{X} f \cdot \overline{f \circ R} d m_{X} \cdot \int_{G \times G} j \otimes \bar{j} d m_{G} \otimes m_{G}=0,
\end{array}
$$

where the last equality follows by the assumption on $j$. The result follows by Theorem 1.1 .

Remark 4.6. The assertion of Proposition 4.5 remains true if in the orthocomplement of $L^{2}\left(X, \mathcal{B}, m_{X}\right) \otimes \mathbb{1}_{G}$ there are finitely many rational eigenvalues (in the proof we need to exclude finitely many $r, s)$.

\subsection{Special case: 2-point extensions of odometers}

We now consider the special case when $G=\mathbb{Z} / 2 \mathbb{Z}$. As an immediate consequence of Theorem 2.4, we obtain the following:

Corollary 4.7. Let $T$ be an odometer and let $\phi, \psi: X \rightarrow \mathbb{Z} / 2 \mathbb{Z}$ be ergodic cocyles. Then, either $T_{\phi}$ and $T_{\psi}$ are isomorphic or they are relatively disjoint over $T$, i.e. $J^{e}\left(T_{\phi}, T_{\psi}\right)=$ $\left\{\widetilde{\left(m_{X}\right)_{R}}: R \in C(T)\right\}$.

Remark 4.8. We give now a direct proof of Corollary 4.7. Fix $\rho \in J^{e}\left(T_{\phi}, T_{\psi}\right)$. We have $\left(T_{\phi} \times T_{\psi}, \rho\right) \simeq\left(T_{\phi \times \psi \circ R}, \kappa\right)$ where $\kappa$ projects on $m_{X}$ and $R \in C(T)$. If $\phi \times \psi \circ R$ is ergodic, it follows by Lemma 2.1 that $\kappa=m_{X} \otimes\left(m_{G} \otimes m_{G}\right)$, so $\rho=\widetilde{\left(m_{X}\right)_{R}}$. If $\phi \times \psi \circ R$ is not ergodic, then

$$
\phi-\psi \circ R=\xi-\xi \circ T \text { for some measurable } \xi: X \rightarrow \mathbb{Z} / 2 \mathbb{Z} .
$$

It follows that $T_{\phi}$ and $T_{\psi}$ are isomorphic: $R_{\xi} \circ T_{\phi}=T_{\psi} \circ R_{\xi}$.

We also have the following (cf. Proposition 4.4). 
Corollary 4.9. Let $T$ be an odometer and let $\psi: X \rightarrow \mathbb{Z} / 2 \mathbb{Z}$ be ergodic. Assume that $T_{\psi}$ has continuous spectrum in the orthocomplement of $L^{2}\left(X, \mathcal{B}, m_{X}\right) \otimes \mathbb{1}_{\mathbb{Z} / 2 \mathbb{Z}}$ and $C\left(T_{\psi}\right)$ is $\mathbb{Z} / 2 \mathbb{Z}$-trivial. Assume, moreover, that $r \neq s$ are prime numbers such that $T^{r}$ and $T^{s}$ are ergodic. Then $\left(T_{\psi}\right)^{r}$ and $\left(T_{\psi}\right)^{s}$ are not isomorphic. form.

Now, using Corollary 4.7, the corresponding part of Proposition 4.5 takes the following

Corollary 4.10. Let $\bar{T}$ be a uniquely ergodic homeomorphism which is a continuous extension of an odometer $T$, measure-theoretically isomorphic to T. Assume that its (rational discrete) spectrum is generated by finitely many prime numbers. Assume that $\psi: X \rightarrow \mathbb{Z} / 2 \mathbb{Z}$ is a cocycle, 10 is satisfied, and $T_{\psi}$ has continuous spectrum in the ortho-

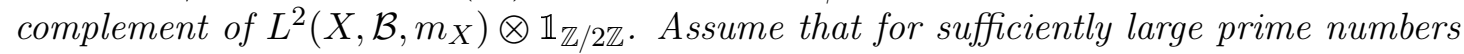
$r \neq s$, the automorphisms $\left(T_{\psi}\right)^{r}$ and $\left(T_{\psi}\right)^{s}$ are not isomorphic. Then for each $f \in C(X)$ and $\mathbb{1} \neq j \in \widehat{\mathbb{Z} / 2 \mathbb{Z}}$, (1) is satisfied for $\bar{T}_{\bar{\psi}}$ and $(f \circ \pi) \otimes j$ at each point.

\section{Applications}

\subsection{Bijective substitutions}

Let $\theta: A \rightarrow A^{\lambda}$ be a bijective substitution with the corresponding bijections $\sigma_{i} \in \mathscr{S}_{r}$. Let $C(\theta)$ denote the centralizer of the set $\left\{\sigma_{i}: i=0, \ldots, \lambda-1\right\}$ in $\mathscr{S}_{r}$. Assume that $\eta \in C(\theta)$. Then $\eta$ induces a map $\widetilde{\eta}$ (both on finite blocks over $A$ and on $A^{\mathbb{Z}}$ ) given by

$$
\widetilde{\eta}(y)[n]:=\eta(y[n]) \text { for each } n \in \mathbb{Z} .
$$

We claim that $\tilde{\eta}(X(\theta))=X(\theta)$. Indeed, since $\eta\left(\sigma_{i}(0)\right)=\sigma_{i}(\eta(0))$, it follows that

$$
\widetilde{\eta}\left(\theta^{n}(0)\right)=\theta^{n}(\eta(0))
$$

and we use the transitivity of the action of the group $G$ generated by $\sigma_{0}, \ldots, \sigma_{\lambda-1}$ on $A$. Since $\widetilde{\eta}$ commutes with the shift, $\widetilde{\eta} \in C(S, X(\theta))$ (indeed, $(S, X(\theta))$ is uniquely ergodic, so $\widetilde{\eta}$ must preserve the unique measure). Now, the result from [34] shows that this is the only way to get non-trivial elements in the centralizer of the (measure-theoretic) dynamical system determined by a bijective substitution:

Theorem 5.1 ([34]). $C(S, X(\theta))=\left\{S^{i} \circ \widetilde{\eta}: i \in \mathbb{Z}, \eta \in C(\theta)\right\}$.

Suppose now that $\theta: G \rightarrow G^{\lambda}$ is a group substitution, i.e. $\theta(g)=\theta(e) \circ g$ for $g \in G$. Notice first that in each column $j$ of the matrix for $\theta$, we have elements $\theta(e)[j] \cdot g$. Therefore

$$
\sigma_{j} \text { is the left translation on } G \text { by } \theta(e)[j]
$$

and the group generated by $\theta(e)[j], j=0, \ldots, \lambda-1$, is $G$. It follows that the group generated by $\sigma_{0}, \ldots, \sigma_{\lambda-1}$ is the group of all left translations on $G$. Its centralizer $C(\theta)$ is equal to the group of all right translations. Thus, we obtain the following consequence of Theorem 5.1: 
Corollary 5.2. The centralizer of the group substitutions is G-trivial.

Remark 5.3. The discrete part of the spectrum of the dynamical system $(S, X(\theta))$, where $\theta$ is a substitution, consists of the spectrum of the underlying odometer and a cyclic group determined by the height $h$ of the substitution [6]. It follows that when the height is equal to 1 , then the spectrum is continuous in the orthocomplement of the $L^{2}$-space of the underlying odometer. Otherwise, in this orthocomplement we have the cyclic group of eigenvalues generated by $e^{2 \pi i / h}$.

We are now ready to show that Sarnak's conjecture holds for dynamical systems given by bijective substitutions.

Theorem 5.4. For each bijective substitution $\theta: A \rightarrow A^{r}$, each function $F \in C(X(\theta))$, each bounded by 1, aperiodic multiplicative function $\boldsymbol{\lambda}: \mathbb{N} \rightarrow \mathbb{C}$ and each $y \in X(\theta)$, we have

$$
\frac{1}{N} \sum_{n \leqslant N} F\left(S^{n} y\right) \boldsymbol{\lambda}(n) \rightarrow 0 \text { when } N \rightarrow \infty
$$

In particular, each topological dynamical system determined by a bijective substitution satisfies Sarnak's conjecture.

Proof. It follows by Lemma 3.19 that it is enough to prove 112 for the dynamical system $(S, X(\bar{\theta}))$ corresponding to the group cover substitution $\bar{\theta}$ of $\theta$. Moreover, in view of Lemma 3.8, we can study instead its topologically isomorphic model $\left(S_{\varphi}, X(\widehat{x}) \times G\right)$.

Fix $f \otimes j$ with $f \in C(X(\widehat{x}))$, where $j \in C(G), \int j d m_{G}=0$. In view of Theorem 1.1. Lemma 3.17, Corollary 5.2 and Proposition 4.5, Remark 4.6 and Remark 5.3, for each $(y, g) \in X(\widehat{x}) \times G$, we have

$$
\frac{1}{N} \sum_{n \leqslant N}(f \otimes j)\left(\left(S_{\varphi}\right)^{n}(y, g)\right) \boldsymbol{\lambda}(n) \rightarrow 0
$$

for each multiplicative function $\boldsymbol{\lambda},|\boldsymbol{\lambda}| \leqslant 1$. If we now fix $\boldsymbol{\lambda}$, then we have the relevant convergence (against this fixed $\boldsymbol{\lambda}$ ) for a linearly dense set of functions in $C(X(\hat{x}) \times G)$, hence for all functions in $C(X(\widehat{x}) \times G)$ and the result follows.

Remark 5.5. Let $j:=\mathbb{1}_{G}$. Using (13), for each $y \in X(\widehat{x})$, we have

$$
\frac{1}{N} \sum_{n \leqslant N} f\left(S^{n} y\right) \boldsymbol{\lambda}(n) \rightarrow 0
$$

for each bounded by 1 , aperiodic multiplicative function $\boldsymbol{\lambda}$. This can be also proved more directly. Notice that for each odometer $(T, X)$ we have (1) true with $\boldsymbol{\mu}$ replaced by $\boldsymbol{\lambda}$ since each finite system enjoys this property and $(T, X)$ is a topological inverse limit of such systems. If $(\bar{T}, \bar{X})$ is a uniquely ergodic topological extension of $(T, X)$, measuretheoretically isomorphic to $\left(T, X, m_{X}\right)$, we can apply Lemma 7 and Proposition 3 in [1] to lift the orthogonality condition $(12)$ from the odometer to $(\bar{T}, \bar{X})$. 
Remark 5.6. The proof of Sarnak's conjecture also gives the following: whenever $(S, X(\theta))$ is a subshift given by a bijective substitution, for each ergodic powers $S^{r}$ and $S^{s}$, each point $(y, z) \in X(\theta) \times X(\theta)$ is generic (for an ergodic measure).

Remark 5.7. In part 5 of [10], Drmota explains how the method developed by Mauduit and Rivat in the proof of Theorem 1 of [38] can be applied to any bijective substitution (Definition 4.1 in [10] of "invertible $\lambda$-automatic sequence" corresponds to our bijective substitution of constant length $\lambda$ ). In particular, Theorem 5.5 of [10] says that any bijective substitution $\theta$ on the alphabet $A$ satisfies a Prime Number Theorem, i.e. that for any $a \in A, \lim _{N \rightarrow \infty} \frac{1}{\pi(N)} \mid\left\{1 \leqslant p<N: p\right.$ is prime, $\left.x_{\theta}[p]=a\right\} \mid$ exists (here $x_{\theta}$ stands for a fixed point given by $\theta$ ). In [38], the proof of Theorem 2 can be deduced from the proof of Theorem 1 just by replacing the classical Vaughan identity by the similar result for the Möbius function (see (13.39) and (13.40) in [21]). It follows from this remark that the arguments given by Drmota in [10] in order to generalize Theorem 1 from [38] to any bijective substitution is still valid to generalize Theorem 2 from 38 to any bijective substitution. This means that if $\theta$ is a bijective substitution and $J: A \rightarrow \mathbb{C}$ then

$$
\frac{1}{N} \sum_{n \leqslant N} J\left(x_{\theta}[n]\right) \boldsymbol{\mu}(n) \rightarrow 0
$$

\subsection{Regular Morse sequences and the Rudin-Shapiro case}

In [31, it has been proved that the centralizer of the dynamical systems given by so called regular Morse sequences [30] $x=b^{0} \times b^{1} \times \ldots\left(b^{t} \in\{0,1\}^{\lambda_{t}}, t \geqslant 0\right)$ is $\mathbb{Z} / 2 \mathbb{Z}$-trivial.

Corollary 5.8. If $x=b^{0} \times b^{1} \times \ldots$ is a regular Morse sequence for which the set $\left\{p: p\right.$ is prime and $p \mid \lambda_{t}$ for some $\left.t\right\}$ is finite. Then (12) holds in the dynamical system given by $x$.

Proof. The result follows from Corollaries 4.9 and 4.10 and the proof of Theorem 5.4 .

In [33, the Rudin-Shapiro type sequences are considered. These are 0-1-sequences $x \in\{0,1\}^{\mathbb{N}}$ such that $x[n]$ is equal to the mod 2 frequency of the block $1 * \ldots * 1$ (with fixed number of $*$ ) in the block given by the binary expansion on $n{ }^{11}$ As shown in [33], the corresponding subshift is given by a Toeplitz type $\mathbb{Z} / 2 \mathbb{Z}$-extension of the dyadic odometer, and the whole method applies.

Corollary 5.9. If $x$ is a Rudin-Shapiro type sequence then 12 holds in $(S, X(x))$. In particular, Sarnak's conjecture holds in the dynamical system given by the classical Rudin-Shapiro sequence.

Proof. Since $(S, X(x))$ has the Lebesgue component of multiplicity $2^{k}$ in the spectrum in the orthocomplement of the space generated by eigenfunctions [33], it follows that its $s$ th and $r$ th power also have Lebesgue components in the spectrum, of multiplicity $s 2^{k}$ and $r 2^{k}$, respectively. Thus, these powers cannot be isomorphic, unless $s=r$. The result follows from Corollary 4.10 .

\footnotetext{
${ }^{11}$ We can also consider $1 * \ldots * 0$.
} 


\section{Spectral approach and other methods}

Let $(S, X)$ with $X \subset A^{\mathbb{Z}}$ be a subshift over a finite alphabet $A$ with $|A|=r \geqslant 2$.

\subsection{First remarks}

Lemma 6.1. Suppose that (1) holds for arbitrary $x \in X$, for each function $f=\mathbb{1}_{B}$, where $B \in A^{k}$ is a block of finite length ( $k \geqslant 1$ is arbitrary) that appears on $X$. Then Sarnak's conjecture holds for $(S, X)$.

Proof. It suffices to show (1) for a linearly dense family of functions in $C(X)$ : e.g. functions which depend on a finite number of coordinates. The space of (continuous) functions depending on coordinates $[-k, k]$ in the full shift has dimension $r^{2 k+1}$, which is at the same time the number of possible blocks of length $2 k+1$. In a similar way, for a subshift, we just need to count the number of distinct $(2 k+1)$-blocks appearing on $X$. Moreover, the family of their characteristic functions is linearly independent.

Remark 6.2. There are other choices of finite families of functions than those in Lemma 6.1 which also yield the validity of Sarnak's conjecture. For example, when $r=2$ we can use the so called Walsh basis: for each $K \geqslant 1$, we consider the characters of the group $\{0,1\}^{2 K+1}: f_{C}(x)=(-1)^{\sum_{i \in C} x[i]}$ for $C \subset\{-K, \ldots, K\}$ and $x \in X \subset A^{\mathbb{Z}}$.

Remark 6.3. In [3, 15] the convergence in (1) is proved at any point for $f(y)=(-1)^{y[0]}$ $\left(f=f_{\{0\}}\right.$ in the notation from Remark 6.2 for Kakutani sequences ${ }^{12}$ A natural question arises whether this is sufficient to obtain Sarnak's conjecture for the corresponding dynamical system. In general, it does not seem to be automatic that (1) for functions depending on one coordinate implies (1) for functions depending on more coordinates. E.g., in [1, where Sarnak's conjecture is proved for the 0-1-subshift generated by the Thue-Morse sequence, (1) for $f(y)=(-1)^{y[0]}$ is proved by completely different methods than for continuous functions invariant under the map $y \mapsto \widetilde{y}$, where $\widetilde{y}[n]=1-y[n]{ }^{13}$ We note that the method from Corollary 4.7 does not apply to Kakutani systems since their centralizer can be uncountable: there are Kakutani sequences for which the corresponding dynamical systems are rigid 32. However, in Section 6.2 we provide an argument which in Section 6.3 will be used to show that [3, 15] yield Sarnak's conjecture for the dynamical systems given by Kakutani sequences.

Remark 6.4. We have already shown that Sarnak's conjecture holds for the dynamical system given by the Rudin-Shapiro sequence, see Corollary 5.9. Recall also that in this case (1) was shown earlier in [38] for $f(y)=(-1)^{y[0]}$ (at any point). Here the situation is more delicate if we want to apply the method from Section 6.2 we need more functions, see Section 6.3 for more details.

\footnotetext{
${ }^{12}$ The uniformity of estimates in these papers yields indeed (1) at any point $y \in X(x)$ ).

${ }^{13}$ E.g. $g(y)=(-1)^{y[0]+y[1]}$ is invariant under this map; notice that $g=f_{\{0,1\}}$ from Remark 6.2 .
} 


\subsection{Spectral approach}

Lemma 6.5. Assume that $T$ is a uniquely ergodic homeomorphism of a compact metric space $X$. Denote the unique T-invariant measure by $\mu$. Assume that the unitary operator $U_{T}: L^{2}(X, \mathcal{B}, \mu) \rightarrow L^{2}(X, \mathcal{B}, \mu), U_{T} g:=g \circ T$, has simple spectrum. Assume that the maximal spectral type of $U_{T}$ is realized by $F \in C(X)$. If $F$ satisfies (1) at each point $x \in X$ then $(T, X)$ satisfies Sarnak's conjecture.

Proof. Observe first that if $F$ satisfies (1) at each point then the same is true for each function $p\left(U_{T}\right) F$ of $F$ (where $p(z)=\sum_{\ell=-K}^{K} a_{\ell} z^{\ell}$ is a trigonometric polynomial). By the simplicity of the spectrum of $U_{T}$, the set of functions of the form $p\left(U_{T}\right) F$ is dense in $L^{2}(X, \mathcal{B}, \mu)$. We now repeat the argument from Lemma 7 in [1]. Fix $G \in C(X), x \in X$ and $\varepsilon>0$. Find a trigonometric polynomial $p$ so that $\left\|p\left(U_{T}\right) F-G\right\|_{2}<\varepsilon$. Let $N_{0}$ be such that for $N \geqslant N_{0},\left|\frac{1}{N} \sum_{n \leqslant N} p\left(U_{T}\right) F\left(T^{n} x\right) \boldsymbol{\mu}(n)\right|<\varepsilon$ for all $N>N_{0}$. Then (since $T$ is uniquely ergodic and $|\boldsymbol{\mu}| \leqslant 1$ )

$$
\begin{aligned}
& \left|\frac{1}{N} \sum_{n \leqslant N} G\left(T^{n} x\right) \boldsymbol{\mu}(n)\right| \\
& \leqslant \frac{1}{N} \sum_{n \leqslant N}\left|\left(G-p\left(U_{T}\right) F\right)\left(T^{n} x\right)\right||\boldsymbol{\mu}(n)|+\left|\frac{1}{N} \sum_{n \leqslant N}\left(p\left(U_{T}\right) F\right)\left(T^{n} x\right) \boldsymbol{\mu}(n)\right| \\
& \leqslant \frac{1}{N} \sum_{n \leqslant N}\left|\left(G-p\left(U_{T}\right) F\right)\left(T^{n} x\right)\right|+\varepsilon \rightarrow\left\|G-p\left(U_{T}\right) F\right\|_{1}+\varepsilon
\end{aligned}
$$

when $N \rightarrow \infty$. Since $\left\|G-p\left(U_{T}\right) F\right\|_{1} \leqslant\left\|G-p\left(U_{T}\right) F\right\|_{2}<\varepsilon$, the result follows.

\section{Remark 6.6.}

(A) The assertion of Lemma 6.5 remains true if we take any bounded arithmetic function $\boldsymbol{\lambda}: \mathbb{N} \rightarrow \mathbb{C}$ instead of $\boldsymbol{\mu}$ (both in (1) and in Sarnak's conjecture). The proof is the same.

(B) Fraczek [12] showed that for each automorphism $T$ on $(X, \mathcal{B}, \mu)$, where $X$ is a compact metric space, the maximal spectral type of $U_{T}$ is always realized by a continuous function. However, in order to prove Sarnak's conjecture using Lemma 6.5, we look for natural continuous functions realizing the maximal spectral type for which we can show that (1) holds.

We would like to mention also an open problem raised by Thouvenot in the 1980th whether each ergodic dynamical system has $L^{1}$-simple spectrum, i.e., for some $f \in L^{1}(X, \mathcal{B}, \mu)$, we have $\operatorname{span}\left\{f \circ T^{k}: k \in \mathbb{Z}\right\}$ dense in $L^{1}(X, \mathcal{B}, \mu)$. If the answer to Thouvenot's problem is positive and the $L^{1}$-simplicity can be realized by a continuous function $f$ in each uniquely ergodic system, then (cf. the proof of Lemma 6.5 to prove Sarnak's conjecture we need to check (1) for $f$ (at each point).

(C) Suppose that the continuous and discrete part of the maximal spectral type of $U_{T}$ are realized by $f \in C(X)$ and $g \in C(X)$, respectively. Then, by elementary spectral 
theory, $F=f+g \in C(X)$ realizes the maximal spectral type of $U_{T}$ and, clearly, it suffices to check that (1) holds both for $f$ and $g$ (at each point) to see that it holds for $F$ (at each point).

(D) Lemma 6.5 has a natural extension to uniquely ergodic homeomorphisms $T$ such that $U_{T}$ has non-trivial multiplicity. All we need to know is that $L^{2}(X, \mathcal{B}, \mu)$ has a decomposition into cyclic spaces: $L^{2}(X, \mathcal{B}, \mu)=\bigoplus_{k \geqslant 1} \mathbb{Z}\left(f_{k}\right)$ with $f_{k} \in C(X)$ and check (1) for these generators ${ }^{14}$ We will find such functions in the next section in case of the dynamical systems given by the Rudin-Shapiro type sequences ${ }^{15}$

\subsection{Applications}

\section{Generalized Morse sequences over $A=\{0,1\}$}

Proposition 6.7. Let $x$ be a generalized Morse sequence over $A=\{0,1\}$. Then Sarnak's conjecture holds for $(S, X(x))$ if and only if (1) holds (at each point) for $f(y)=(-1)^{y[0]}$.

Proof. Recall that $(S, X(x))$ is uniquely ergodic [26] (with the unique invariant measure $\mu_{x}$ ) and has simple spectrum [29]. As proved in [29] (see also [17]) $f(y)=(-1)^{y[0]}$ realizes the continuous part of the maximal spectral type of $U_{S}$ (on $L^{2}\left(X(x), \mu_{x}\right)$ ). Moreover, the discrete part is given by the equicontinuous factor of $(S, X(x))$, which is the odometer determined by $\lambda_{t}, t \geqslant 0$. It follows that the eigenfunctions $g_{i}, i \geqslant 1$, are continuous. If $g=\sum_{i \geqslant 1} a_{i} g_{i}$, each $a_{i} \neq 0, \sum_{i \geqslant 1}\left|a_{i}\right|<+\infty$, then $g$ is a continuous function realizing the discrete part of the maximal spectral type of $U_{S}$. Since each odometer is a topological inverse limit of systems defined on finitely many points, and for finite systems Sarnak's conjecture holds because of the PNT in arithmetic progressions, therefore $g$ satisifes (1). Thus, in view of Lemma 6.5 and Remark 6.6 (C), it suffices to prove (1) for $f$ to obtain the validity of Sarnak's conjecture.

Corollary 6.8. Sarnak's conjecture holds for the dynamical systems given by Kakutani sequences.

Proof. In view of the above proposition, it suffices to prove (1) for $f$ which was done in [3, 15] (cf. Remark 6.3).

Rudin-Shapiro type sequences Recall that the classical Rudin-Sharpiro sequence $x \in\{0,1\}^{\mathbb{N}}$ is defined in the following way:

- take the fixed point of the substitution $a \mapsto a b, b \mapsto a c, c \mapsto d b$ and $d \mapsto d c$,

\footnotetext{
${ }^{14}$ Recall however that it is open whether for an arbitrary automorphism $T$ on $(X, \mathcal{B}, \mu)$, where $X$ is a compact metric space there are continuous functions $f_{k}, k \geqslant 1$ such that $L^{2}(X, \mathcal{B}, \mu)=\bigoplus_{k \geqslant 1} \mathbb{Z}\left(f_{k}\right)$ and $\sigma_{f_{k+1}} \ll \sigma_{f_{k}}, k \geqslant 1$, see e.g. 25] for more details.

${ }^{15}$ Recall that in the general case of dynamical systems given by the Rudin-Shapiro type sequences, this multiplicity is of the form $2^{k}, k \geqslant 1[33$.
} 
- use the code $a, b \mapsto 0$ and $c, d \mapsto 1$ to pass to the space of 0-1-sequences (the map arising from this code yields a topological isomorphism of the relevant subshifts on four and two letters).

The multiplicity of the corresponding dynamical system on the continuous part of the spectrum is equal to 2 [33, 43]. It follows from [33] and Remark 6.6 (D) that, in order to obtain Sarnak's conjecture for the corresponding subshift, we need to check (1) for two continuous functions $f \cdot \mathbb{1}_{D_{0}^{2}}$ and $f \cdot \mathbb{1}_{D_{1}^{2}}$ (cf. Remark 3.12). It follows immediately from the definition of $D_{0}^{2}$ and the recognizability of substitutions [41] that $\mathbb{1}_{D_{0}^{2}}$ is a continuous function depending on a finite number of coordinates. Therefore, to obtain Sarnak's conjecture for the subshift given by the Rudin-Shapiro sequence, we would have to check (1) for the elements of the Walsh basis of order $4 M$, where $M$ is the constant of recognizability, see Remark 3.14. Recall that (1) was already shown for $f$ in [38, 46]. Notice that this approach to prove Sarnak's conjecture is completely different from the one presented in the preceding sections (cf. Corollary 5.9). The above applies to all Rudin-Shapiro type sequences.

\subsection{Comparison with results of Veech [47]}

In the recent preprint [47, Veech considers a class of systems for which Sarnak's conjecture holds. We will now briefly present his work and then compare it with our results. Assume that $\lambda_{n} \geqslant 2$ for $n \geqslant 0$, then set $n_{0}:=1$ and $n_{t}:=\prod_{k=0}^{t-1} \lambda_{k}, t \geqslant 1$ and define

$$
X:=\operatorname{liminv}_{t \rightarrow \infty} \mathbb{Z} / n_{t} \mathbb{Z}=\left\{x=\left(x_{t}\right): 0 \leqslant x_{t}<n_{t}, x_{t+1}=x_{t} \bmod n_{t}, t \geqslant 1\right\} .
$$

This is a compact, Abelian, monothetic group on which we consider $T x=x+\theta$ with $\theta=(1,1, \ldots)$. It is not hard to see that the systems obtained this way are naturally isomorphic to the odometers considered in Section 3.1. The sequence of towers $\mathcal{D}^{t}, t \geqslant 1$, in the new coordinates is determined by

$$
D_{0}^{t}:=\left\{x \in X: x_{t}=0\right\}
$$

and we obtain pairwise disjoint sets $D_{0}^{t}, T D_{0}^{t}, \ldots T^{n_{t}-1} D_{0}^{t}$ with $\bigcup_{j=0}^{n_{t}-1} T^{j} D_{0}^{t}=X$. Then define

$$
\tau(x):=\min \left\{t \geqslant 1: x_{t} \neq n_{t}-1\right\} .
$$

We have $\lim _{x \rightarrow-\theta} \tau(x)=\infty$ and $\tau$ is continuous on $X \backslash\{-\theta\}$.

Let $K$ be a compact group and take $(\Psi(t))_{t \geqslant 1} \subset K$. Set

$$
f(x):=\Psi(\tau(x)) .
$$

Then $\Psi$ is locally constant on $X \backslash\{-\theta\}$ and $f \in C(X \backslash\{-\theta\}, K)$. There are some assumptions on the sequence $\Psi$ made in [47]:

(i) $\lim _{t \rightarrow \infty} \Psi(t)$ does not exist,

(ii) $\{\Psi(t): t \geqslant 1\}$ generates a dense subgroup of $K$, and so does the set $\left\{\Psi(t) \Psi(u)^{-1}\right.$ : $t, u \geqslant 1\}$, 
(iii) $(\Psi(t))_{t \geqslant 1}$ is recurrent (that is, every initial block of $\Psi$ repeats infinitely often).

Remark 6.9. If $K=\mathbb{Z} / 2 \mathbb{Z}$, the conditions (ii) and (iii) are not necessary.

Let $M_{\Psi} \subset K^{\mathbb{Z}}$ be the closure of all sequences $\left(f(x+n \theta)_{n \in \mathbb{Z}}\right.$ for $x \in X \backslash \mathbb{Z} \theta$. On $M_{\Psi}$, we consider the usual shift $S$. Let

$$
m: M_{\Psi} \rightarrow K \text { be given by } m(y)=y[0] .
$$

Finally, let $S_{m}: M_{\Psi} \times K \rightarrow M_{\Psi} \times K$ be the skew product defined as $S_{m}(y, k)=$ $(S y, m(y) k)$. Then $S_{m}$ is a homeomorphism of $M_{\Psi} \times K$.

Theorem 6.10 ([47]). Suppose additionally that the set $\left\{\lambda_{t}: t \geqslant 0\right\}$ is finite. Then, under the above assumptions, $\left(S_{m}, M_{\Psi} \times K\right)$ satisfies Sarnak's conjecture.

Remark 6.11. It is not hard to see that in the language of [7], the function $f$ is a semicocycle over an odometer (that is, a function continuous on a residual subset of an odometer). It follows from [7] that the dynamical system given by $\left(S, M_{\Psi}\right)$ is a Toeplitz dynamical system (cf. Section 3.1). The system is regular [7, hence uniquely ergodic and measure-theoretically isomorphic to the odometer $(T, X)$.

Notice that if $x_{t}=n_{t}-1$ then also $x_{k}=n_{k}-1$ for $1 \leqslant k \leqslant t-1$. It follows immediately that $\tau$ is constant on each $D_{i}^{t}, 0 \leqslant i \leqslant n_{t}-2$. Therefore, the cocycle $f: X \rightarrow K$ defined above is a Morse cocycle (cf. Section 3.1). It has the following additional property:

$$
f \text { is constant on } \bigcup_{1 \leqslant j \leqslant \lambda_{t+1}-1} D_{j n_{t}-1}^{t+1} \text {. }
$$

Condition (14) yields the class of Morse sequences $x=b^{0} \times b^{1} \times \ldots$, where each block $b_{t}$, $t \geqslant 0$, is of the form $b^{t}=e k \ldots k^{\left|b^{t}\right|-1}$ (cf. Remark 3.9). In particular, if $K=\mathbb{Z} / 2 \mathbb{Z}$, we have $x=b^{0} \times b^{1} \times \ldots$, where $b^{t}=0 \ldots 0, b^{t}=01 \ldots 01$ or $b^{t}=010 \ldots 10, t \geqslant 0$. Notice that Kakutani sequences are of this form.

Notice also that $\left(S_{m}, M_{\Psi} \times K\right)$ corresponds to $\left(S_{\varphi}, X(\widehat{x}) \times G\right)$ defined in Section 3.1 which is, in turn, topologically isomorphic to $(S, X(x))$.

It follows that Theorem 6.10 is a significant extension of the validity of Sarnak's conjecture for Kakutani systems (cf. Corollary 6.8) to general compact groups.

\section{References}

[1] E. H. El Abdalaoui, S. Kasjan, And M. Lemańczyk, 0-1 sequences of the Thue-Morse type and Sarnak's conjecture. To appear in Proc. Amer. Math. Soc.

[2] E. H. El Abdalaoui, M. Lemańczyk, and T. De la Rue, On spectral disjointness of powers for rank-one transformations and Möbius orthogonality, J. Funct. Anal., 266 (2014), pp. $284-317$.

[3] J. Bourgain, Möbius-Walsh correlation bounds and an estimate of Mauduit and Rivat, J. Anal. Math., 119 (2013), pp. 147-163. 
[4] - On the correlation of the Moebius function with rank-one systems, J. Anal. Math., 120 (2013), pp. 105-130.

[5] J. Bourgain, P. Sarnak, And T. Ziegler, Disjointness of Möbius from horocycle flows, in From Fourier analysis and number theory to Radon transforms and geometry, vol. 28 of Dev. Math., Springer, New York, 2013, pp. 67-83.

[6] F. M. DekKIng, The spectrum of dynamical systems arising from substitutions of constant length, Z. Wahrscheinlichkeitstheorie und Verw. Gebiete, 41 (1977/78), pp. 221-239.

[7] T. Downarowicz, Survey of odometers and Toeplitz flows, in Algebraic and topological dynamics, vol. 385 of Contemp. Math., Amer. Math. Soc., Providence, RI, 2005, pp. 7-37.

[8] T. Downarowicz and S. KaSJan, Odometers and Toeplitz subshifts revisited in the context of Sarnak's conjecture. Preprint.

[9] T. Downarowicz, J. Kwiatkowski, and Y. Lacroix, Spectral isomorphisms of Morse flows, Fund. Math., 163 (2000), pp. 193-213.

[10] M. DRMota, Subsequences of automatic sequences and uniform distribution, in Uniform distribution and quasi-Monte Carlo methods, vol. 15 of Radon Ser. Comput. Appl. Math., De Gruyter, Berlin, 2014, pp. 87-104.

[11] S. Ferenczi And C. Mauduit, On Sarnak's conjecture and Veech's question for interval exchanges. Preprint.

[12] K. FrączeK, On a function that realizes the maximal spectral type, Studia Math., 124 (1997), pp. 1-7.

[13] H. Furstenberg, Recurrence in ergodic theory and combinatorial number theory, Princeton University Press, Princeton, N.J., 1981. M. B. Porter Lectures.

[14] E. Glasner, Ergodic theory via joinings, vol. 101 of Mathematical Surveys and Monographs, American Mathematical Society, Providence, RI, 2003.

[15] B. Green, On (not) computing the Möbius function using bounded depth circuits, Combin. Probab. Comput., 21 (2012), pp. 942-951.

[16] B. Green and T. TaO, The Möbius function is strongly orthogonal to nilsequences, Ann. of Math. (2), 175 (2012), pp. 541-566.

[17] M. Guenais, Morse cocycles and simple Lebesgue spectrum, Ergodic Theory Dynam. Systems, 19 (1999), pp. 437-446.

[18] F. Hahn AND W. PARRY, Some characteristic properties of dynamical systems with quasidiscrete spectra, Math. Systems Theory, 2 (1968), pp. 179-190.

[19] A. J. HARPER, A different proof of a finite version of Vinogradov's bilinear sum inequality. https://www.dpmms.cam.ac.uk/ $a j h 228 /$ FiniteBilinearNotes.pdf.

[20] J. L. Herning, Spectrum and Factors of Substitution Dynamical Systems, ProQuest LLC, Ann Arbor, MI, 2013. Thesis (Ph.D.)-The George Washington University. 
[21] H. Iwaniec and E. Kowalski, Analytic number theory, vol. 53 of American Mathematical Society Colloquium Publications, American Mathematical Society, Providence, RI, 2004.

[22] K. Jacobs and M. Keane, 0-1-sequences of Toeplitz type, Z. Wahrscheinlichkeitstheorie und Verw. Gebiete, 13 (1969), pp. 123-131.

[23] T. Kamae, A topological invariant of substitution minimal sets, J. Math. Soc. Japan, 24 (1972), pp. 285-306.

[24] I. Kátai, A remark on a theorem of H. Daboussi, Acta Math. Hungar., 47 (1986), pp. 223225.

[25] A. Katok and M. LemańczyK, Some new cases of realization of spectral multiplicity function for ergodic transformations, Fund. Math., 206 (2009), pp. 185-215.

[26] M. Keane, Generalized Morse sequences, Z. Wahrscheinlichkeitstheorie und Verw. Gebiete, 10 (1968), pp. 335-353.

[27] J. Kulaga-Przymus and M. Lemańczyk, Ergodic sequences along which Sarnak's conjecture holds for uniquely ergodic flows. In preparation.

[28] - The Moebius function and continuous extensions of rotations. http://arxiv.org/ abs/1310.2546, 102013.

[29] J. Kwiatkowski, Spectral isomorphism of Morse dynamical systems, Bull. Acad. Polon. Sci. Sér. Sci. Math., 29 (1981), pp. 105-114.

[30] _ Isomorphism of regular Morse dynamical systems, Studia Math., 72 (1982), pp. 59-89.

[31] M. Lemańczyk, The centralizer of Morse shifts, Ann. Sci. Univ. Clermont-Ferrand II Probab. Appl., (1985), pp. 43-56.

[32] — The rank of regular Morse dynamical systems, Z. Wahrsch. Verw. Gebiete, 70 (1985), pp. 33-48.

[33] — Toeplitz $Z_{2}$-extensions, Ann. Inst. H. Poincaré Probab. Statist., 24 (1988), pp. 1-43.

[34] M. Lemańczyk and M. K. Mentzen, On metric properties of substitutions, Compositio Math., 65 (1988), pp. 241-263.

[35] J. Liu and P. Sarnak, The Möbius function and distal flows, Duke Math. J., 164 (2015), pp. 1353-1399.

[36] J. Mathew And M. G. NADKARni, A measure preserving transformation whose spectrum has Lebesgue component of multiplicity two, Bull. London Math. Soc., 16 (1984), pp. $402-$ 406.

[37] C. Mauduit and J. Rivat, Sur un problème de Gelfond: la somme des chiffres des nombres premiers, Ann. of Math. (2), 171 (2010), pp. 1591-1646.

[38] _ Prime numbers along Rudin-Shapiro sequences. To appear in Journal of the European Math. Soc. http://iml.univ-mrs.fr/〜rivat/preprints/PNT-RS.pdf, 2013. 
[39] M. K. Mentzen, Invariant sub- $\sigma$-algebras for substitutions of constant length, Studia Math., 92 (1989), pp. 257-273.

[40] — Ergodic properties of group extensions of dynamical systems with discrete spectra, Studia Math., 101 (1991), pp. 19-31.

[41] B. Mossé, Properties of words and recognizability of fixed points of a substitution, Theor. Comput. Sci., 99 (1992), pp. 327-334.

[42] W. ParrY, Topics in ergodic theory, vol. 75 of Cambridge Tracts in Mathematics, Cambridge University Press, Cambridge, 2004. Reprint of the 1981 original.

[43] M. QuefFÉLEC, Substitution dynamical systems-spectral analysis, vol. 1294 of Lecture Notes in Mathematics, Springer-Verlag, Berlin, second ed., 2010.

[44] E. A. Robinson, JR., Spectral multiplicity for nonabelian Morse sequences, in Dynamical systems (College Park, MD, 1986-87), vol. 1342 of Lecture Notes in Math., Springer, Berlin, 1988, pp. 645-652.

[45] P. SARnak, Three lectures on the Möbius function, randomness and dynamics. http: //publications.ias.edu/sarnak/

[46] T. TAO. http://mathoverflow.net/questions/97261/mobius-randomness-of-therudin-shapiro-sequence.

[47] W. A. Veech, Möbius orthogonality for generalized Morse-Kakutani flows. Preprint.

[48] _ Strict ergodicity in zero dimensional dynamical systems and the Kronecker-Weyl theorem mod 2, Trans. Amer. Math. Soc., 140 (1969), pp. 1-33.

[49] _ Ergodic theory and uniform distribution, in Journées Arithmétiques de Luminy (Colloq. Internat. CNRS, Centre Univ. Luminy, Luminy, 1978), vol. 61 of Astérisque, Soc. Math. France, Paris, 1979, pp. 223-234.

Sébastien Ferenczi

Institut de Mathématiques de Marseille, CNRS - UMR 7373

Case 907 - 163, av. de Luminy, F13288 Marseille Cedex 9, France

E-mail address: ssferenczi@gmail.com

Joanna Kułaga-Przymus

Institute of Mathematics, Polish Acadamy of Sciences, Śniadeckich 8, 00-956 Warszawa, POLAND

Faculty of Mathematics and Computer Science, Nicolaus Copernicus University, Chopina 12/18, 87-100 Toruń, Poland

E-mail address: joanna.kulaga@gmail.com

Mariusz Lemańczyk

Faculty of Mathematics and Computer Science, Nicolaus Copernicus University, Chopina 12/18, 87-100 TORuń, Poland

E-mail address: mlem@mat.umk.pl

Christian Mauduit

Institut de Mathématiques de Marseille, CNRS - UMR 7373

Case 907 - 163, av. de Luminy, F13288 Marseille Cedex 9, France

E-mail address: mauduit@iml.univ-mrs.fr 